\title{
Co-Ni-Zn Ferrites Fabricated by Spark Plasma Sintering
}

\author{
Andrea Balla', János Moczó², Zoltán Károly²* \\ ${ }_{1}^{1}$ Doctoral School on Materials Sciences and Technologies, Óbuda University, H-1034 Budapest, Bécsi str. 96/B, Hungary \\ 2 Research Centre for Natural Sciences, Hungarian Academy of Sciences, H-1117 Budapest, Magyar tudósok krt. 2, Hungary \\ *Corresponding author, e-mail: karoly.zoltan@ttk.mta.hu
}

Received: 18 July 2019, Accepted: 29 October 2019, Published online: 23 December 2019

\begin{abstract}
$\mathrm{Ni}_{0,4} \mathrm{CO}_{0,2} \mathrm{Zn}_{0,4} \mathrm{Fe}_{2} \mathrm{O}_{4}$ spinel ferrites have been synthesized by precipitation method from an aqueous solution and bulk samples were fabricated by Spark Plasma Sintering (SPS) to investigate the microstructure and the mechanical properties. Although SPS is considered as a rapid compaction technique, its application is uncommon for ferrites due to reactions occurring between the graphite die and the ferrite powder at elevated temperature. In our tests this problem was circumvented by an alumina film applied on the die. We found that both chemical and phase composition could be retained in the sintered specimens after sintering. In addition, they exhibited improved mechanical properties in terms of hardness (10 GPa) and fracture toughness $\left(2.7 \mathrm{MPa} \cdot \mathrm{m}^{-1 / 2}\right)$ as compared to conventionally sintered reference samples.
\end{abstract}

Keywords

ferrites, morphology, Spark Plasma Sintering

\section{Introduction}

Spinel ferrites are soft magnetic materials that found widespread application in areas such as medical diagnostics, high density information storage systems, ferro fluid technology, gas sensors, microwave and electronic devices/transformers, inductors, choke coils, etc. [1-3]. $\mathrm{Ni}$-Zn ferrites show outstanding performance in high frequency applications by virtue of their high resistance, low eddy current losses combined with high Curie temperature, permeability and permittivity $[4,5]$. The performance of ferrites is basically affected by its chemical composition and microstructure as well, which in turn are greatly influenced by the fabrication method. The conventional sintering process consists of long heat treatment at high temperature over $1100{ }^{\circ} \mathrm{C}$. At such high temperature volatile zinc component may be partially evaporated that eventually reduces resistivity $[6,7]$. In addition, long sintering time may also lead to grain coarsening and inhomogeneous microstructure that has adverse effect for the electromagnetic performance. In parallel, the microstructure also influences the mechanical properties of the ceramic ferrites. Similar to other ceramics, ferrites possess low fracture toughness. There are several approaches to improve the mechanical properties of ceramics, a great part of which consist of using additives including small metal or ceramic particles, whiskers, fibers, etc. that prevent propagation of cracks by various mechanisms [8]. The incorporation of a second phase into a ferrite matrix, however, may induce undesirable reactions in between that could ruin the chemical composition and consequently the electronic performance of the particular ferrite. On the other hand, the mechanical properties can be also improved by a better control of the microstructure of the sintered bulk material. According to the HallPetch relation, a microstructure having smaller grains improves the mechanical properties. For certain applications such as in microwave devices nanometric sized ferrite grains could be of great advantage, while in other application e.g. for power ferrites the grain size beyond the optimal $2-10 \mu \mathrm{m}$ is not favorable. Nevertheless, by simply retaining the original dimensions of the fine particles in the final microstructure and preventing grain growth during sintering, one can improve the mechanical properties, as well. In contrast to conventional sintering, Spark Plasma Sintering (SPS) technique offers a rapid fabrication method under controlled atmosphere and typically at reduced temperature $[9,10]$ as compared to conventional sintering technique. Owing to the very short holding time at the high temperature of sintering that is typical for SPS, grain coarsening can be avoided [11]. However, up to now quite a few studies have been reported on the 
SPS sintering of ferrites [12-16]. This is because in SPS technique the powder is consolidated in graphite moulds that create strong reducing conditions for ferrites $[12,13]$. To prevent partial or complete reduction of the metal-ions, short sintering time and low sintering temperatures (500$600{ }^{\circ} \mathrm{C}$ ) are applied [14-16]. Although this technique results in consolidated bulk material with good magnetic properties, the attained lower relative densities imply inferior mechanical properties that may present drawback for application point of view.

In this work, we report on the SPS sintering of cobalt doped Ni-Zn ferrite. We investigated the obtained microstructure and the mechanical properties of the sintered ferrite specimen. To prevent reaction between the graphite mould and the ferrite powder the graphite dies were covered with an inert alumina layer.

\section{Experimental}

\subsection{Synthesis of ferrite powder}

Ferrite powder was synthesized by co-precipitation method using aqueous solutions of analytical grade reagents of Nickel (II) nitrate hexahydrate $\left[\mathrm{Ni}\left(\mathrm{NO}_{3}\right)_{2} \cdot 6 \mathrm{H}_{2} \mathrm{O}\right]$, Zinc nitrate hexahydrate $\left[\mathrm{Zn}\left(\mathrm{NO}_{3}\right)_{2} \cdot 6 \mathrm{H}_{2} \mathrm{O}\right]$, Cobalt (II) nitrate hexahydrate $\left[\mathrm{Co}\left(\mathrm{NO}_{3}\right)_{2} \cdot 6 \mathrm{H}_{2} \mathrm{O}\right]$ (Alfa Aesar), Iron (III) nitrate nonahydrate $\left[\mathrm{Fe}\left(\mathrm{NO}_{3}\right)_{3} \cdot 9 \mathrm{H}_{2} \mathrm{O}\right]$ (Molar Chemicals Kft.) and sodium carbonate $\left[\mathrm{Na}_{2} \mathrm{CO}_{3}\right]$ (SISECAM). The aqueous solution of precipitating reagent $\left[\mathrm{Na}_{2} \mathrm{CO}_{3}\right]$ was added drop-wise into the nitrate solutions under continuous stirring until reaching $\mathrm{pH}$ 9. Stirring was continued for an additional hour. The precipitated grains were collected, washed with distilled water to remove the residue salts until reaching $\mathrm{pH} 7$, then dried at $80^{\circ} \mathrm{C}$. The dried powder was calcined at $1100{ }^{\circ} \mathrm{C}$ for 2 hours. Before sintering the powder was dry ball milled for 1 hour to disintegrate the agglomerates.

\subsection{Sintering}

Bulk sintered specimens of $3 \mathrm{~cm}$ in diameter and $5 \mathrm{~mm}$ in thickness were fabricated from the as-prepared ferrite powder by Spark Plasma Sintering apparatus (HD P5, FCT GmbH). Samples were sintered at four different temperatures as $1000,1100,1200$ and $1300^{\circ} \mathrm{C}$ using a holding time of 5 minutes in each test. Heating rate was invariably $100{ }^{\circ} \mathrm{C} / \mathrm{min}$. During sintering a constant uniaxial pressure of $30 \mathrm{MPa}$ was applied.

As a reference, conventional sintered specimens of similar dimensions were also prepared using $30 \mathrm{MPa}$ pressure for compaction before sintering. The graphite parts were covered with thin alumina film that hindered direct contact between the graphite die and the powder components to avoid any possible undesirable reactions in between.

\subsection{Characterization}

The particle-size distribution of the synthesized ferrite powder was analysed by laser scattering method (Partica LA-960, Horiba) after 120 seconds of ultrasonic agitation without the use of any flocculants. Chemical composition was determined by energy dispersive X-ray fluorescence method (Niton XL3t Thermo Fisher Scientific). Morphology and microstructure of the ferrite powder and the sintered samples were characterized by scanning electron microscopy (SEM, LEO $1540 \mathrm{XB}$ with a heated field emission tungsten cathode) and by X-ray diffraction (XRD) performed on a Bruker AXD 8D using $\mathrm{CuK} \alpha 1$ radiation. The bulk density of the sintered samples was determined on the basis of the Archimedes method. Microhardness was measured by Vickers indentation method [HV0.2 $(2 \mathrm{~N} ; 10 \mathrm{~s})]$ and given as an average of 10 measurements on different locations of the particular specimens. The small size of the specimen did not allow performing a standard fracture toughness test. Instead, it was calculated according to the Shetty formula [17]:

$K_{I C}=0.0899\left(\frac{H P}{4 l}\right)^{0.5}$

where $H$ stands for the hardness, $P$ is the indentation load and $l$ is the length of the indentation crack.

\section{Results and discussion}

The particle-size distribution of the synthesized particles is shown in Fig. 1. The powder has a relatively narrow size distribution ranges from $1 \mu \mathrm{m}$ to $15 \mu \mathrm{m}$ with a mean size of $7 \mu \mathrm{m}$. SEM image of (Fig. 2) the synthesized ferrite particles, however, reveals that larger particles are typically consisted of aggregated fine particles, that could be hardly disintegrated by ultrasonic treatment.

Application of agglomerated powder is not favored in conventional sintering process, since it prevents attaining

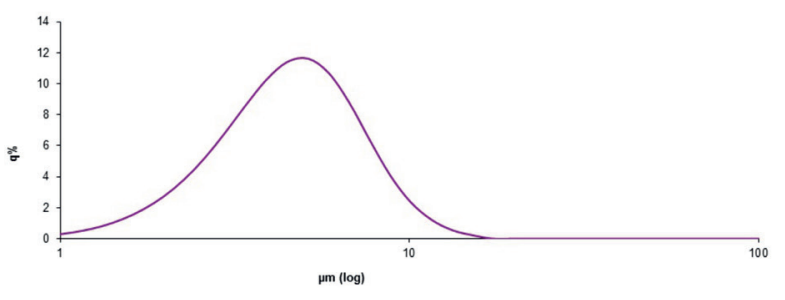

Fig. 1 Size distribution of the synthesized ferrite particles 


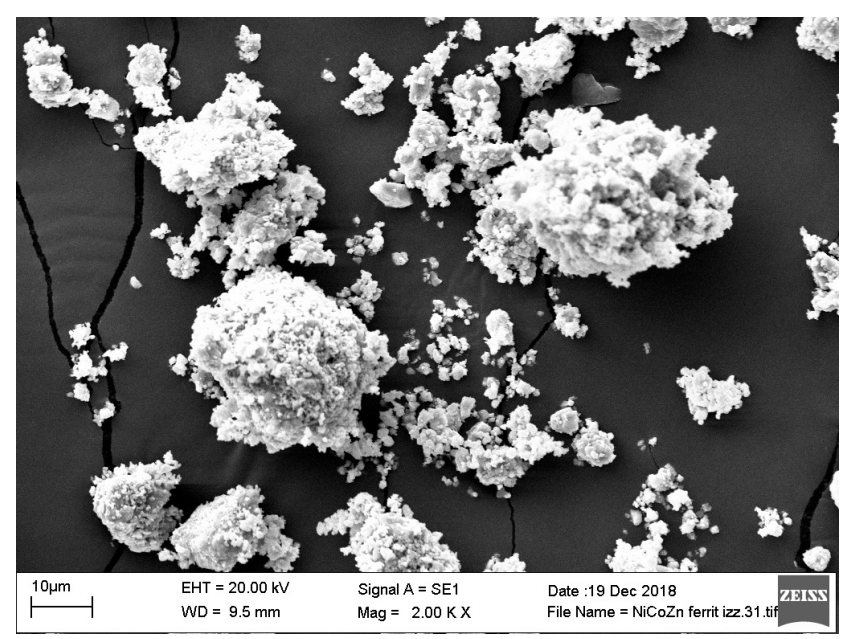

Fig. 2 SEM image of the synthesized ferrite particles

higher green density that ultimately may lead to increased porosity in the final product resulting in inferior mechanical properties. In contrast, SPS technique is less sensitive for the powder morphology because of the constantly applied pressure during sintering. The major sintering parameters are plotted in Fig. 3 with respect to elapsed time.

Chemical analysis showed that the exact stochiometry of the synthesized ferrite particles was $\mathrm{Ni}_{0.39} \mathrm{Co}_{0.22} \mathrm{Zn}_{0.39} \mathrm{Fe}_{2} \mathrm{O}_{4}$. The XRD patterns of the synthesized powder as well as the SPS sintered samples (Fig. 4) show sharp peaks indicating completely crystallized particles. All the peaks can be assigned to the complex ferrite [18] with no indication of separated binary or mono metal oxides.

Fig. 5 compares the relative density values of the sintered specimens as a function of sintering temperatures for conventional and Spark Plasma Sintering. The density increased with the sintering temperature for both methods. However, the relative density of SPS sintered specimens tends to be higher as compared to those of the conventional sintered specimens.

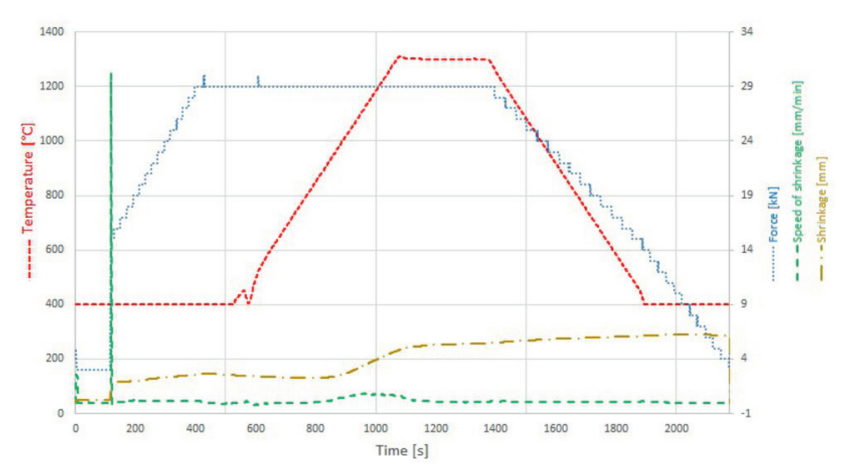

Fig. 3 Major parameters of ferrite Spark Plasma Sintering in the function of time

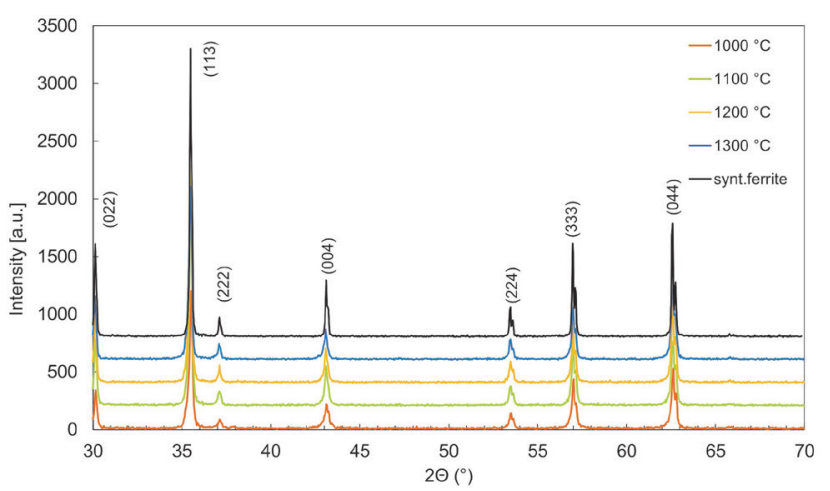

Fig. 4 XRD patterns of the synthesized ferrites and bulk specimens sintered at different temperatures

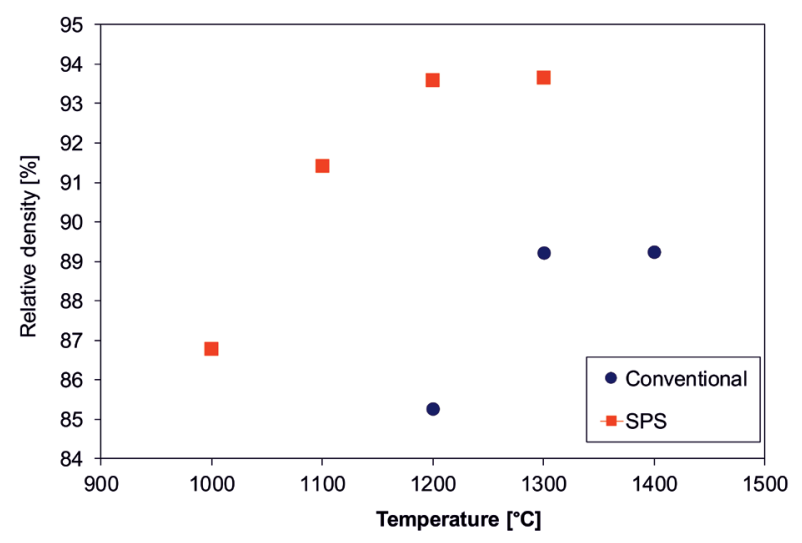

Fig. 5 Relative densities of the ferrite specimens with respect to sintering temperature

A ca. $94 \%$ maximum value of relative densities was achieved at $1200{ }^{\circ} \mathrm{C}$ for the SPS samples that did not increase further at still higher sintering temperature. In addition, above $1300{ }^{\circ} \mathrm{C}$, there is a risk of $\mathrm{Zn}$ losses due to partial or even complete evaporation [6], which make no sense of raising the temperature further. For all the sintered specimens we performed elemental analysis and found that the composition was identical to that of the synthesized starting powder. It confirmed that employing rapid sintering technique the original composition can be retained up to $1300^{\circ} \mathrm{C}$.

Fig. 6 (a)-(d) shows the scanning images of the fractured surfaces of ferrites sintered by SPS at various temperatures. Minor porosity can be observed only for the sample sintered at the lowest applied temperature (Fig. 6 (a)). There is a noticeable difference in the grain size of the samples sintered at different temperatures, which became larger with increasing temperature. While the size of the grains was around $4-7 \mu \mathrm{m}$ at 1000 and $1100^{\circ} \mathrm{C}$, it grew further up to $10-20 \mu \mathrm{m}$ above $1200{ }^{\circ} \mathrm{C}$ sintering temperature. Grain coarsening is a typical phenomenon in conventional sintering, where the large grains are growing 


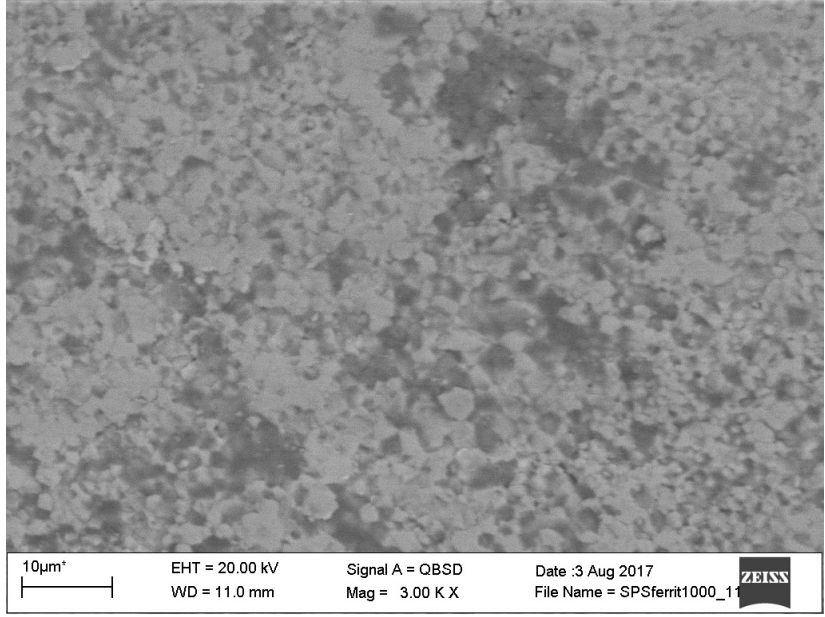

(a)

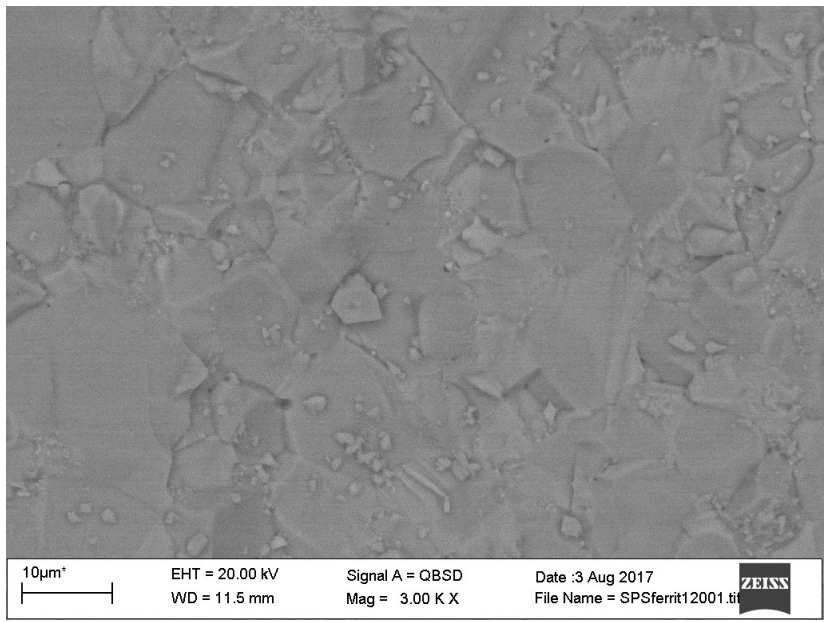

(c)

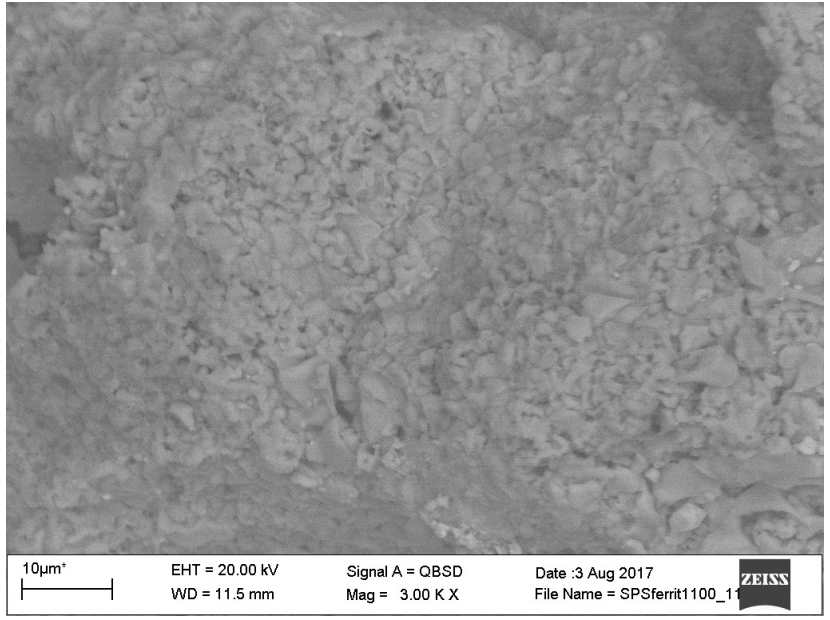

(b)

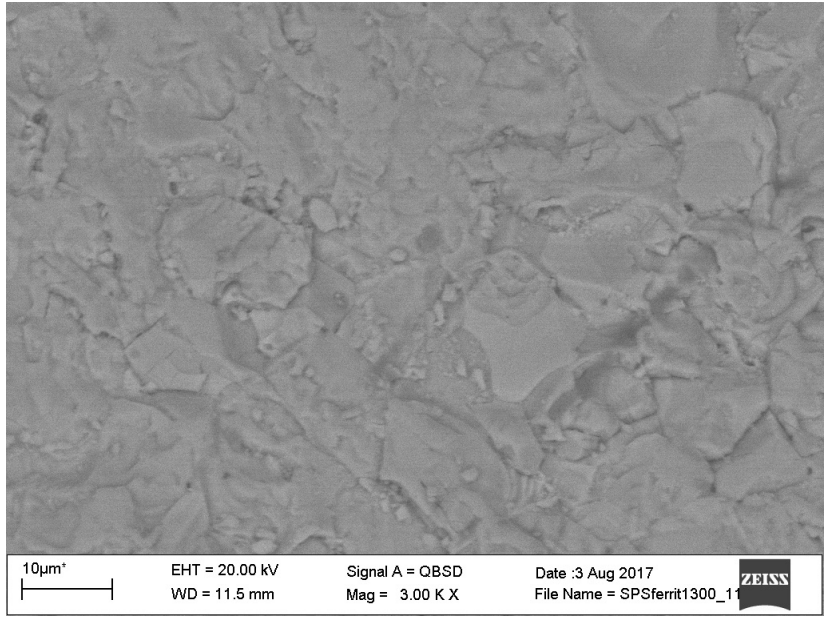

(d)

Fig. 6 Scanning images of spark plasma ferrites sintered at: (a) $1000{ }^{\circ} \mathrm{C}$, (b) $1100{ }^{\circ} \mathrm{C}$, (c) $1200{ }^{\circ} \mathrm{C}$, and (d) $1300{ }^{\circ} \mathrm{C}$

on the expense of the smaller ones, but it was not expected for SPS sintering due to the applied very short holding time. While the occurred grain coarsening implies rapid diffusion processes during sintering, the remained porosity suggests insufficient sintering time. However, longer sintering time probably gave rise to even larger grains that would not be beneficial for the mechanical properties.

\subsection{Mechanical properties}

The mean values of measured Vicker's microhardness with respect to sintering temperature were plotted on Fig. 7. It can be observed that values range from 8 to $10 \mathrm{GPa}$ that is a little higher as compared to 6-8 GPa typical for ferrites sintered by the conventional technique. Considering that the trend of hardness commonly follows the density one would expect higher hardness with increasing temperature. At the same time, however, another phenomenon, the grain coarsening takes place, too. It is well known, described by the Hall-Petch relation, that finer grains results in greater hardness and vice versa. The two phenomena probably compensated each other that ultimately led to invariability in the hardness.

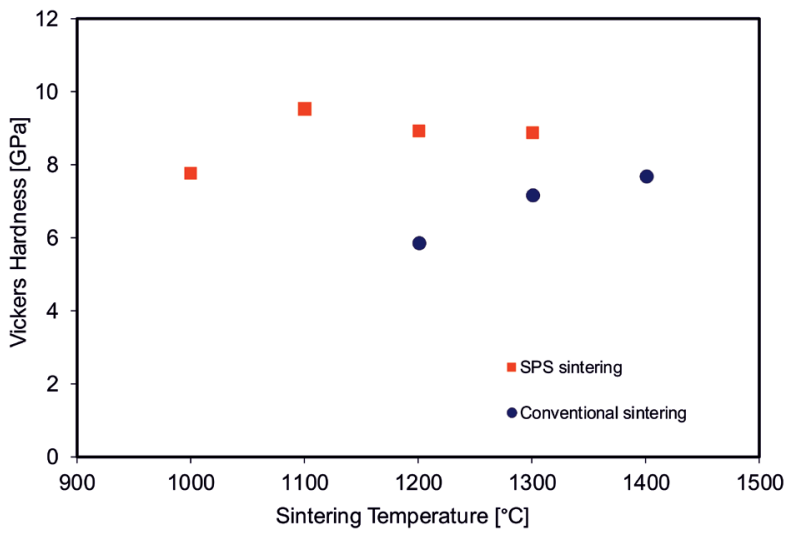

Fig. 7 The Vickers hardness of the conventionally and spark plasma sintered specimens in the function of the sintering temperatures 
In contrast to hardness, fracture toughness showed an increasing trend with respect to the sintering temperature similarly to the density values (Fig. 8). It is reasoned by the fact that unlike hardness, fracture toughness is much less affected by the grain size. However, comparing the fracture toughness of the samples sintered at $1000{ }^{\circ} \mathrm{C}$ and $1300{ }^{\circ} \mathrm{C}$ a much higher, ca. $70 \%$ improvement could be observed as compared to relative density. The achieved higher $K_{I C}$ values of $2.5 \mathrm{MPam}^{-1 / 2}$ is also significantly higher as compared with ferrites sintered by conventional $\left(0.85 \mathrm{MPam}^{-1 / 2}\right)$ or other special $\left(1.24 \mathrm{MPam}^{-1 / 2}\right)$ method $[19,20]$.

\section{Conclusion}

The mechanical properties of $\mathrm{Ni}_{0,4} \mathrm{Co}_{0,2} \mathrm{Zn}_{0,4} \mathrm{Fe}_{2} \mathrm{O}_{4}$ ferrite specimen fabricated by Spark Plasma Sintering technique at various temperatures without using any sintering additives was studied. A thin alumina film was applied around the sample to prevent any reaction between the graphite die and the ferrite. We found that the hardness of the sintered ferrites slightly increased with the sintering temperature and invariably exceeded that of the reference samples sintered in conventional way. Fracture toughness of the SPS sintered

\section{References}

[1] Ramesh, S., Chandra Sekhar, B., Subba Rao, P. S. V., Parvatheeswara Rao, B. "Microstructural and magnetic behavior of mixed $\mathrm{Ni}-\mathrm{Zn}-\mathrm{Co}$ and $\mathrm{Ni}-\mathrm{Zn}-\mathrm{Mn}$ ferrites", Ceramics International, 40(6), pp. 8729-8735, 2014.

https://doi.org/10.1016/j.ceramint.2014.01.092

[2] Wang, S. F., Hsu, Y. F., Chou, K. M., Tsai, J. T. "Effects of co-dopants on the magnetic properties of Ni-Zn ferrites", Journal of Magnetism and Magnetic Materials, 374, pp. 402-410, 2015. https://doi.org/10.1016/j.jmmm.2014.08.075

[3] Ghodake, J. S., Shinde, T. J., Patil, R. P., Patil, S. B., Suryavanshi, S. S. "Initial permeability of Zn-Ni-Co ferrite", Journal of Magnetism and Magnetic Materials, 378, pp. 436-439, 2015.

https://doi.org/10.1016/j.jmmm.2014.11.041

[4] Cullity, B. D., Graham, C. D. "Introduction to magnetic materials", John Wiley \& Sons, Inc., Hoboken, NJ, USA, 2009.

https://doi.org/10.1002/9780470386323

[5] Atif, M., Nadeem, M. "Sol-gel synthesis of nanocrystalline $\mathrm{Zn}_{1-\mathrm{x}} \mathrm{Ni}_{\mathrm{x}} \mathrm{Fe}_{2} \mathrm{O}_{4}$ ceramics and its structural, magnetic and dielectric properties", Journal of Sol-Gel Science and Technology, 72(3), pp. $615-626,2014$.

https://doi.org/10.1007/s10971-014-3484-4

[6] Verma, A., Goel, T. C., Mendiratta, R. G., Gupta, R. G. "High-resistivity nickel-zinc ferrites by the citrate precursor method", Journal of Magnetism and Magnetic Materials, 192(2), pp. 271-276, 1999.

https://doi.org/10.1016/S0304-8853(98)00592-7

[7] Valenzuela, R. "Magnetic ceramics", Cambridge University Press, Cambridge, UK, 1994.

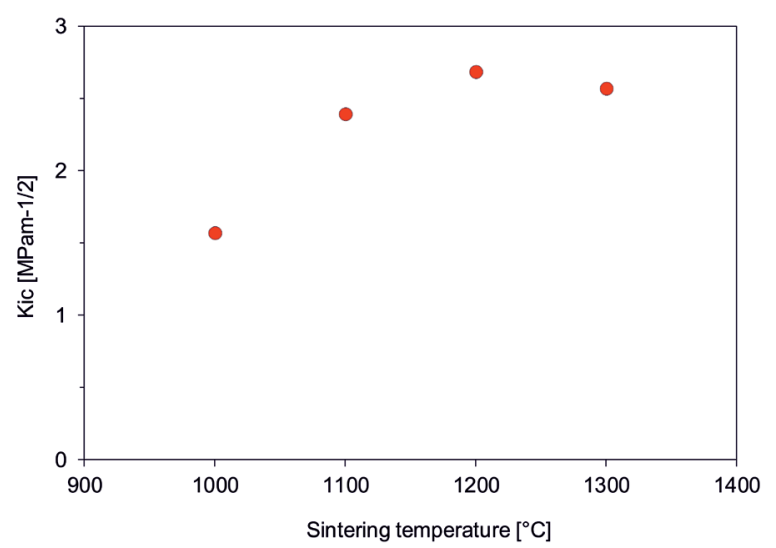

Fig. 8 Effects of the temperature on the fracture toughness of the spark plasma sintered specimens

ferrites was significantly higher as compared to reference material and increased with rising sintering temperature reaching a maximum of $2.7 \mathrm{MPa} \cdot \mathrm{m}^{-1 / 2}$ at $1200^{\circ} \mathrm{C}$. Our findings showed that spinel ferrites, presumably regardless of their composition can be compacted by SPS technique gaining improved mechanical properties.

[8] Okada, A. "Ceramic-Matrix Composites", In: Somiya, S., Aldinger, F., Claussen, N., Kaneno, M., Koumoto, K., Spriggs, R. M., Uchino, K. (eds.) Handbook of Advanced Ceramics, Elsevier Inc. Academic Press, London, UK, 2003, pp. 417-445. https://doi.org/10.1016/B978-012654640-8/50042-0

[9] Aubert, A., Loyau, V., Mazaleyrat, F., LoBue, M. "Uniaxial anisotropy and enhanced magnetostriction of $\mathrm{CoFe}_{2} \mathrm{O}_{4}$ induced by reaction under uniaxial pressure with SPS", Journal of the Eurpean Ceramic Society, 37(9), pp. 3101-3105, 2017. https://doi.org/10.1016/j.jeurceramsoc.2017.03.036

[10] Mercier, A., Chaplier, G., Pasko, A., Loyau, V., Mazaleyrat, F. "Cobalt Doping Effect on Ni-Zn-Cu Ferrites Produced by Reactive Sintering", Physics Procedia, 75, pp. 1306-1313, 2015. https://doi.org/10.1016/j.phpro.2015.12.146

[11] Munir, Z. A., Anselmi-Tamburini, U., Ohyanagi, M. "The effect of electric field and pressure on the synthesis and consolidation of materials: A review of the spark plasma sintering method", Journal of Materials Science, 41(3), pp. 763-777, 2006. https://doi.org/10.1007/s10853-006-6555-2

[12] Valenzuela, R., Gaudisson, T., Ammar, S. "Severe reduction of $\mathrm{Ni}-\mathrm{Zn}$ ferrites during consolidation by Spark Plasma Sintering (SPS)", Journal of Magnetism and Magnetic Materials, 400, pp. 311-314, 2016. https://doi.org/10.1016/j.jmmm.2015.07.044

[13] Yamamoto, S., Horie, S, Tanamachi, N., Kurisu, H., Matsuura, M. "Fabrication of high-permeabilityferrite by spark-plasma-sintering method", Journal of Magnetism and Magnetic Materials, 235(1-3), pp. 218-222, 2001. https://doi.org/10.1016/S0304-8853(01)00341-9 
[14] Ortega-Zempoalteca, R., Flores-Arias, Y., Vázquez-Victorio, G., Gaudisson, T., Ammar, S., Vargas-Osorio, Z., Acevedo-Salas, U., Valenzuela, R. "The effects of spark plasma sintering consolidation on the ferromagnetic resonance spectra (FMR) of Ni-Zn ferrites", Physica Status Solidi A, 211(5), pp. 1062-1066, 2014. https://doi.org/10.1002/pssa.201300762

[15] Cruz-Franco, B., Gaudisson, T., Ammar, S., Bolarín-Miró, A. M., Sánchez de Jesús, F., Mazaleyrat, F., Nowak, S., VázquezVictorio, G., Ortega-Zempoalteca, R., Valenzuela, R. "Magnetic Properties of Nanostructured Spinel Ferrites", IEEE Transactions on Magnetics, 50(4), Article number: 2800106, 2014. https://doi.org/10.1109/TMAG.2013.2283875

[16] Valenzuela, R., Beji, Z., Herbst, F., Ammar, S. "Ferromagnetic resonance behavior of spark plasma sintered $\mathrm{Ni}-\mathrm{Zn}$ ferrite nanoparticles produced by a chemical route", Journal of Applied Physics, 109, Article number: 07A329, 2011. https://doi.org/10.1063/1.3565397

[17] Kovalčíková, A., Balko, J., Balázsi, C., Hvizdoš, P., Dusza, J. "Influence of hBN content on mechanical and tribological properties of $\mathrm{Si}_{3} \mathrm{~N}_{4} / \mathrm{BN}$ ceramic composites", Journal of the European Ceramic Society, 34(14), pp. 3319-3328, 2014. https://doi.org/10.1016/j.jeurceramsoc.2014.02.021
[18] Xie, J. L., Han, M., Chen, L., Kuang, R., Deng, L. "Microwaveabsorbing properties of NiCoZn spinel ferrites", Journal of Magnetism and Magnetic Materials, 314(1), pp. 37-42, 2007. https://doi.org/10.1016/j.jmmm.2007.02.124

[19] Gutiérrez-López, J., Levenfeld, B., Várez, A., Pastor, J. Y., Cañadas, I., Rodríguez, J. "Study of the densification, mechanical and magnetic properties of $\mathrm{Ni}-\mathrm{Zn}$ ferrites sintered in a solar furnace", Ceramics International, 41(5), pp. 6534-6541, 2015. https://doi.org/10.1016/j.ceramint.2015.01.096

[20] Gutiérrez-López, J., Rodriguez-Senín, E., Pastor, J. Y., Paris, M. A., Martín, A., Levenfeld, B., Várez, A. "Microstructure, magnetic and mechanical properties of $\mathrm{Ni}-\mathrm{Zn}$ ferrites prepared by powder injection moulding", Powder Technology, 210(1), pp. 29-35, 2011. https://doi.org/10.1016/j.powtec.2011.02.008 